areas co-registered with VH-IVUS and compared to contrast attenuation to create contrast ratios for each plaque component. These ratios were used to create a colour map of the plaque based on the HU of its constituents and used to test: (A) Classification of plaque components against histology in 10 post-mortem human coronary arteries. (B) Quantification of plaque geometry and composition compared with VH-IVUS in 30 coronary segments. (C) Ability to differentiate 63 patients prospectively enrolled with either stable angina or acute coronary syndrome.

Results (A) CT contrast ratio defined HU-colour maps were created for the 10 post-mortem arteries which were then sectioned into eighty-seven $400 \mu \mathrm{m}$ segments for histological analysis. The maps permitted detection of significant atherosclerosis with sensitivity$92 \%$ and specificity- $90 \%$, calcified-plaque with sensitivity- $80 \%$ and specificity- $88 \%$ and necrotic core sensitivity- $55 \%$, specificity- $96 \%$. If only necrotic core area $>2 \mathrm{~mm}^{2}$ are considered (above the spatial resolution of CT) there is a significant improvement in sensitivity$75 \%$. (B) Plaque-maps were created for $900 \mathrm{~mm}$ of coronary segments and co-registered with VH-IVUS. On average, CT overestimated total plaque area by $44 \%$, vessel volume- $33 \%$, lumen- $10 \%$, necrotic core$140 \%$, fibrous plaque- $70 \%$ and calcified plaque- $9 \%$. However, correlation between CT and VH-IVUS was highly significant $(p<0.001)$ for all measurements: vessel volume $(r=0.86)$, lumen $(r=0.74)$, necrotic core $(r=0.47)$, fibrous plaque $(r=0.74)$ and calcified plaque $r=0.69)$. (C) Culprit lesions of 31 patients with stable angina and 32 with troponin-positive ACS underwent CT prior to PCI. Features discriminating acute from stable plaque detected using the plaquemaps include: micro-calcification- $63 \%$ vs $35 \% \quad(p=0.03)$, distinct necrotic core- $56 \%$ vs $23 \%(p<0.01)$ (Abstract 095 figure 1 ) and positive vessel remodelling- $68 \%$ vs $26 \%(p<0.001)$. The percentage of necrotic core (low attenuation plaque) was higher in acute plaques$54 \%$ vs $44 \%(p<0.01)$ while conversely the percentage of calcified plaque (high attenuation plaque) was lower- $4 \%$ vs $15 \%(p<0.01)$. Intra-plaque contrast was more common $44 \%$ vs $6 \%(p<0.001)$ with high specificity for acute plaques (94\%) and we feel it may represent visualisation of plaque rupture (Abstract 095 figure 1).
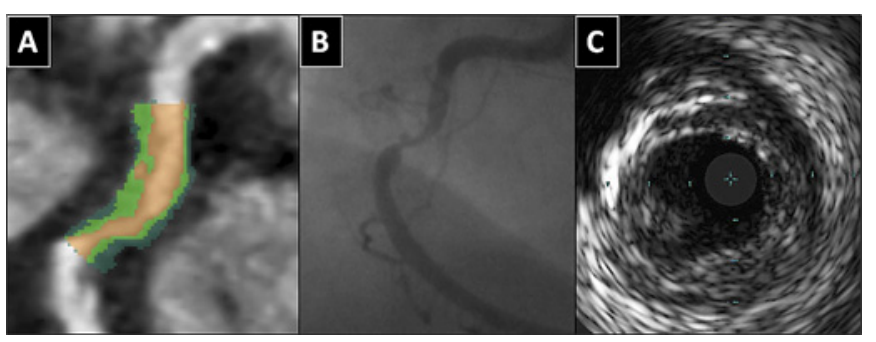

Abstract 095 Figure 1 (A) CT image of intra-plaque contrast with colour mapping. (B) Corresponding coronary angiogram. (C) IVUS reveals plaque rupture at this point.

Conclusion Plaque-mapping with contrast ratios allows plaque quantification and may assist diagnosis of acute plaque rupture.

\section{A COMPARATIVE STUDY OF STANDARD FILTERED BACK PROJECTION WITH NOVEL ITERATIVE RECONSTRUCTION TECHNIQUES IN CARDIAC CT}

doi:10.1136/heartjnl-2012-301877b.96

${ }^{1} \mathrm{P}$ McKavanagh, ${ }^{*}{ }^{1} \mathrm{~L}$ Lusk, ${ }^{1} \mathrm{P}$ Ball, ${ }^{2} \mathrm{M}$ Harbinson, ${ }^{1} \mathrm{~T}$ Trinnick, ${ }^{1} \mathrm{E}$ Duly, ${ }^{1} \mathrm{G}$ Walls, ${ }^{1} \mathrm{~S}$ McCusker, ${ }^{1} \mathrm{C}$ L McQuillan, ${ }^{1} \mathrm{~S}$ Shevlin, ${ }^{1} \mathrm{M}$ Alkhalil, ${ }^{1} \mathrm{P} \mathrm{M}$ Donnelly. ${ }^{1}$ Ulster Hospital, Belfast, UK; ${ }^{2}$ Royal Victoria Hospital, Belfast, UK

Background Iterative reconstruction (IR) is a novel but significant development in CT image acquisition. There have been a number of studies that have reported on the potential of IR in cardiac CT. These retrospectively applied IR in the image domain to images acquired with standard filtered back projection (FBP) techniques This study was part of an ongoing randomised control trial [ISRCTN52480460] evaluating the cost effectiveness of cardiac CT. Methods 250 patients were prospectively enrolled to have a cardiac CT for the investigation of stable chest pain. Written and informed consent was obtained. Data acquisition were performed on a Philips Brilliance 64 . The patients were divided into two groups. Cohort A underwent standard FBP imaging, and Cohort B underwent IR with Idose ${ }^{\circledR}$ (Philips, Cleveland, Ohio, USA). Within each cohort the scan parameters ( $\mathrm{kv}, \mathrm{mAs}$, pitch) and reconstruction protocols (prospective or retrospective) were determined by patient characteristics. Images were assessed for noise and signal quality within regions of interest (ROI) on axial images, and subjectively for image quality by two experienced readers. Noise was defined as the SD of the measured $\mathrm{HU}$, and signal as the HU mean attenuation value. The ROIs were in the ascending aorta, interventricular septum and left ventricular cavity. Subjective image quality was rated blindly using a 5-point Likert scale. Effective radiation dose (ED) of each CTCA was estimated by multiplying the dose-length product by a chest-specific conversion coefficient $\left(\kappa=0.014 \mathrm{mSv} \times \mathrm{mGy}^{-1} \times \mathrm{cm}^{-1}\right)$.

Results Of the 250 patients enrolled 3 withdrew. 146 of the 247 subjects were male with a mean age of 57.93 (SD 9.93). Cohort A consisted of 124 patients, and cohort B 123, with no significant difference in baseline demographics. The mean dose of all FBP was $6.09 \mathrm{mSv}$, (SD 3.16) compared to an IR mean of $4.23 \mathrm{mSv}$, (SD 2.01) which was a dose saving of $1.86 \mathrm{mSv}(30.54 \%)$. This was a significant dose reduction ( $p$ value $<0.0001$.) Mean image quality score obtained from the IR images was 3.67 (SD 1.04) compared to the FBP images of 3.29 (SD 1.17) p value of 0.0067 . There was good agreement between the readers $-\kappa$ coefficient 0.83 . Cohort A consisted of 74 retrospective images and 50 prospective. Cohort $B$ had 116 with retrospective and 7 with prospective. The mean ED for a prospective FBP was $3.50 \mathrm{mSv}$ (SD 1.15), with the IR equivalent being $2.00 \mathrm{mSv}$ (0.72), giving a mean dose saving of $1.50 \mathrm{mSv}(42.86 \%)$. The mean $\mathrm{ED}$ for FBP retrospective studies was $7.85 \mathrm{mSv}$ (SD 2.87), with the IR equivalent being $4.36 \mathrm{mSv}$ (SD 1.99), with a mean dose saving of $3.49 \mathrm{mSv}$ (44.46\%). There was no statistical difference in noise or mean attenuation between the IR and FBP images in all three areas of interest Abstract 096 table 1.

Abstract 096 Table 1

\begin{tabular}{|c|c|c|c|c|c|c|}
\hline \multirow[b]{2}{*}{$\begin{array}{l}\text { Region of } \\
\text { interest }\end{array}$} & \multicolumn{3}{|l|}{ Image noise } & \multicolumn{3}{|l|}{ Attenuation } \\
\hline & FBP & IR & $\begin{array}{l}\mathbf{p} \\
\text { Value }\end{array}$ & FBP & IR & $\begin{array}{l}\text { p } \\
\text { Value }\end{array}$ \\
\hline $\begin{array}{l}\text { Ascending } \\
\text { aorta }\end{array}$ & $29.76 \pm 32.00$ & $27.33 \pm 10.10$ & 0.42 & $505.85 \pm 95.64$ & $520.72 \pm 103.07$ & 0.24 \\
\hline $\begin{array}{l}\text { Interventricular } \\
\text { septum }\end{array}$ & $28.96 \pm 9.63$ & $28.27 \pm 7.53$ & 0.53 & $154.76 \pm 35.28$ & $153.63 \pm 32.41$ & 0.79 \\
\hline Left ventricle & $29.78 \pm 9.36$ & $28.55 \pm 12.39$ & 0.38 & $464.27 \pm 92.50$ & $484.07 \pm 99.38$ & 0.11 \\
\hline
\end{tabular}

Conclusions To our knowledge this is the first study to prospectively compare FBP with IR. It suggests that cardiac IR protocols confer a substantial radiation dose reduction without a compromise in diagnostic quality.

\section{CALCIUM SCORES ARE MORE COST EFFECTIVE FOR RISK STRATIFICATION THAN NICE'S MODIFIED DIAMOND FORRESTER CALCULATOR}

doi:10.1136/heartjnl-2012-301877b.97

${ }^{1} \mathrm{~S}$ McCusker, ${ }^{1} \mathrm{P}$ McKavanagh, ${ }^{*}{ }^{1} \mathrm{~L}$ Lusk, ${ }^{2} \mathrm{~A}$ Agus, ${ }^{1} \mathrm{P}$ Ball, ${ }^{3} \mathrm{M}$ Harbinson, ${ }^{1} \mathrm{~T}$ Trinnick, ${ }^{1} \mathrm{E}$ Duly, ${ }^{1} \mathrm{G}$ Walls, ${ }^{1} \mathrm{~S}$ Shevlin, ${ }^{1} \mathrm{C} \mathrm{L}$ McQuillan, ${ }^{1} \mathrm{M}$ Alkhalil, ${ }^{1} \mathrm{P}$ Donnelly. ${ }^{1}$ Ulster Hospital, Belfast, UK; ${ }^{2}$ CRSC, Glasgow, UK; ${ }^{3}$ Royal Victoria Hospital, Belfast, UK

Background In March 2010 NICE published clinical guideline 95 (CG95). This proposed a move to a primary imaging strategy for 